\title{
The group reduction for bounded cosine functions on UMD spaces
}

\author{
Markus Haase
}

Received: 19 September 2007 / Accepted: 25 April 2008 / Published online: 30 May 2008

(C) The Author(s) 2008

\begin{abstract}
It is shown that if $A$ generates a bounded cosine operator function on a UMD space $X$, then $i(-A)^{1 / 2}$ generates a bounded $C_{0}$-group. The proof uses a transference principle for cosine functions.
\end{abstract}

Keywords Cosine function · Transference principle $\cdot C_{0}$-semigroup $\cdot$ Group • Functional calculus $\cdot$ UMD space $\cdot$ Fattorini's theorem

Mathematics Subject Classification (2000) $\quad 47 \mathrm{~A} 60 \cdot$ 47D06

\section{Introduction}

A cosine function on a (complex) Banach space $X$ is a strongly continuous mapping Cos : $\mathbb{R} \longrightarrow \mathcal{L}(X)$ that satisfies the identity

$$
\operatorname{Cos}(t+s)+\operatorname{Cos}(t-s)=2 \operatorname{Cos}(t) \operatorname{Cos}(s) \quad(t, s \in \mathbb{R})
$$

as well as $\operatorname{Cos}(0)=I$. One can prove from this that a cosine function is exponentially bounded, i.e.,

$$
\theta(\operatorname{Cos}):=\inf \left\{\omega \geq 0 \mid \exists M \geq 1:\|\operatorname{Cos}(t)\| \leq M \mathrm{e}^{\omega|t|}, t \in \mathbb{R}\right\}<\infty .
$$

The generator of a cosine function $\operatorname{Cos}$ is defined as the unique operator $A$ such that

$$
\lambda R\left(\lambda^{2}, A\right)=\int_{0}^{\infty} \mathrm{e}^{-\lambda t} \operatorname{Cos}(t) \mathrm{d} t \quad(\lambda>\theta(\operatorname{Cos})) .
$$

M. Haase ( $\square)$

Delft Institute of Applied Mathematics, Technical University Delft,

PO Box 5031, 2600 GA Delft, The Netherlands

e-mail: m.h.a.haase@tudelft.nl 
The generator $A$ is densely defined, and the cosine function provides solutions to the second-order abstract Cauchy problem

$$
u^{\prime \prime}(t)=A u, \quad u(0)=x, \quad u^{\prime}(0)=0 .
$$

Conversely, if the abstract second order problem for an operator is well-posed, then it gives rise to a cosine function. In this way, cosine functions play the same role for the second order problem as semigroups do for the first order problem. We refer to [1, Chaps. 3.14-3.16] for details on these facts and more material on the theory of cosine functions.

An important example of a cosine function arises as

$$
\operatorname{Cos}(t)=\frac{1}{2}(U(t)+U(-t)) \quad(t \in \mathbb{R}),
$$

where $U$ is a $C_{0}$-group. The generator $A$ of the cosine function and the generator $B$ of the group are then related by $A=B^{2}$. (As an example consider $B=\mathrm{d} / \mathrm{d} t$ the generator of the shift group on $\mathbf{L}^{2}(\mathbb{R})$; then $A$ is the one-dimensional Laplacian.) It is natural to ask, which cosine functions arise in this manner, but in general there is little hope. Indeed, there is no way in general to reconstruct a group $U$ from its associated cosine function. Taking squares deletes information that cannot be recovered. However, as $B$ is a square root of $A$, one might look at $i(-A)^{1 / 2}$, whenever $-A$ is sectorial. (The minus sign is natural here, since the spectrum of $A$ extends to the left.) Let us call the group generated by $i(-A)^{1 / 2}$, if it exists, the square root reduction group associated with the original cosine function. It turns out that in general the square root reduction group does not exist, but only due to a shortcoming of the Banach space. Indeed, Fattorini [8] has shown the following result.

Theorem (Fattorini 1969) Let $A$ be the generator of a cosine function on a UMD space X. If $-A$ is also sectorial, then $i(-A)^{1 / 2}$ generates a $C_{0}$-group.

A proof adapted from the original one is in [1, 3.16.7]. More recently, functional calculus methods have been used to give a different proof (see [11] and combine it with [10, Proposition 3.17] and standard perturbation). However, the approach was via the so-called phase space and a direct functional calculus proof is still to be found.

There is another issue here. Fattorini's theorem is qualitative in nature and tells us nothing about the growth properties of the reduction group, depending on the growth of the cosine function. In particular, it has been an open problem for a long time whether the reduction group associated with a bounded cosine function is itself bounded. On Hilbert spaces this is known to be true [9], but the methods used are typical for Hilbert spaces, finding selfadjointness by introducing an equivalent scalar product. To the best of our knowledge, the last serious attempt to solve the problem on a general UMD space was made by Cioranescu and Keyantuo [5], building on the paper [13]. The present paper solves the problem in the affirmative.

Theorem 1.1 Let A generate a bounded cosine function on a UMD space. Then $i(-A)^{1 / 2}$ generates a bounded $C_{0}$-group.

To avoid many minus signs, it is convenient to change notation a little and write $A$ instead of $-A$. Moreover, we shall prove Theorem 1.1 in two steps according to the following equivalent version:

Theorem 1.1 (Alternative Version) Let $-A$ be the generator of a bounded cosine function Cos on a UMD space $X$. Then there exists a bounded $C_{0}$-group $U$ such that 


$$
\operatorname{Cos}(s)=\frac{1}{2}(U(s)+U(-s)) \quad(s \in \mathbb{R})
$$

and $-i A^{1 / 2}$ is the generator of $U$.

The purpose of this reformulation is to avoid the theory of sectorial operators as long as possible. In fact, our proof of the first part (the existence and boundedness of the group) is essentially self-contained, whereas for the second part (the identification of the generator) one has to appeal to the well-established theory of fractional powers. However, we find it very instructive that this theory is not needed to obtain the existence and boundedness of the group reduction in the first place. Needless to say that we will not make use of Fattorini's original theorem at any point.

The paper is structured as follows. In Sect. 2 we construct a functional calculus $\Phi$ in a Phillips type manner using integrals of the form

$$
T_{\mu} x:=\int_{\mathbb{R}} \operatorname{Cos}(s) x \mu(\mathrm{d} s) \quad(x \in X)
$$

where $\mu$ is a bounded measure. We show how this functional calculus can be interpreted in a canonical way as a functional calculus for a certain unbounded operator $B$. The group $U$ will be given as $U(s)=\Phi\left(e^{-i s \cdot}\right)=e^{-i s B}, s \in \mathbb{R}$, and the (uniform) boundedness of these operators is reduced to the uniform boundedness of certain approximants. By means of a transference principle, which we state and prove in Sect. 3, one reduces this uniform boundedness to the uniform boundedness of certain Fourier-multipliers on the space $\mathbf{L}^{2}(\mathbb{R} ; X)$. In Sect. 4 we briefly provide the necessary background on Fourier multipliers and the notion of UMD spaces, and finally bring the different ingredients together to prove that the group $U$ is uniformly bounded. In Sect. 5 we give a new and simpler proof of a known representation formula for the operators $A^{1 / 2} \operatorname{Sin}(s), s \in \mathbb{R}$, where $(\operatorname{Sin}(s))_{s \in \mathbb{R}}$ is the associated sine function (Theorem 5.1). Again using transference, this leads to an alternative proof of (the first part of) Theorem 1.1. Finally, in Sect. 6 we prove that the operator $B$ constructed before is identical to the square root $A^{1 / 2}$ obtained from $A$ by means of the sectorial functional calculus (or by the classical theory of fractional powers). Moreover, we show that the functional calculus $\Phi$ is compatible with and provides a proper extension of the sectorial functional calculus for $A^{1 / 2}$.

\section{Definitions and conventions}

We usually consider (unbounded) closed operators $A, B$ on a complex Banach space $X$. By $\mathcal{L}(X)$ we denote the set of all bounded (fully defined) operators on $X$. The domain and the range of a general operator $A$ are denoted by $\mathcal{D}(A)$ and $\mathcal{R}(A)$, respectively. Its resolvent is $R(\lambda, A)=(\lambda-A)^{-1}$, and $\varrho(A)$ denotes the set of $\lambda \in \mathbb{C}$ where $R(\lambda, A) \in \mathcal{L}(X)$. Its complement $\sigma(A)=\mathbb{C} \backslash \varrho(A)$ is the spectrum.

For $\omega \in(0, \pi]$ we denote by

$$
S_{\omega}:=\{z \neq 0|| \arg z \mid<\omega\}
$$

the horizontal sector of angle $2 \omega$, symmetric about the positive real axis, and we let $\mathbb{R}_{+}:=$ $[0, \infty)$ the positive semi-axis, including the point 0 . For each open subset $\Omega \subset \mathbb{C}$ we denote by $H^{\infty}(\Omega)$ the Banach algebra of bounded holomorphic functions on $\Omega$. If $\Omega$ is an arbitrary locally compact space, then the set of complex regular Borel measures on $\Omega$ is denoted by $\mathbf{M}(\Omega)$. The Fourier transform of a tempered distribution $\Phi$ on $\mathbb{R}$ is denoted by $\mathcal{F}(\Phi)$ or $\widehat{\Phi}$. We often write $s$ and $t$ (in the Fourier image) to denote the real coordinate, e.g., $\sin t / t$ 
denotes the function $t \longmapsto \sin t / t$. On a complex domain we use $z$ as the coordinate, so that $f(z)$ denotes the function $z \longmapsto f(z)$.

Let $X$ be a Banach space and $p \in[1, \infty)$. For a finite measure $v \in \mathbf{M}(\mathbb{R})$ we denote by

$$
L_{v}:=(f \longmapsto f * v): \mathbf{L}^{\boldsymbol{p}}(\mathbb{R} ; X) \rightarrow \mathbf{L}^{\boldsymbol{p}}(\mathbb{R} ; X)
$$

the convolution operator on the $X$-valued $\mathbf{L}^{p}$-space.

\section{A functional calculus}

We suppose in this section that $-A$ is the generator of a bounded cosine function $(\operatorname{Cos}(t))_{t \in \mathbb{R}}$ on the Banach space $X$. Note that as mentioned in the introduction we consider $-A$ instead of $A$ in order to avoid many minus signs later. In order to construct the reduction group, we shall built up a functional calculus for an yet unknown operator $B$ and only later (Sect. 6) we will identify this operator as $A^{1 / 2}$.

The so-called Phillips calculus for Cos is the mapping

$$
\left(\mu \longmapsto T_{\mu}\right): \mathbf{M}(\mathbb{R}) \longrightarrow \mathcal{L}(X)
$$

with $T_{\mu}$ being defined by

$$
T_{\mu} x:=\int_{\mathbb{R}} \operatorname{Cos}(s) x \mu(\mathrm{d} s) \quad(x \in X, \mu \in \mathbf{M}(\mathbb{R})) .
$$

Since Cos is an even function, the operator $T_{\mu}$ depends only on the even part $\mu_{e}$ of $\mu$, defined by

$$
\mu_{e}(A):=\frac{1}{2}(\mu(A)+\mu(-A)) \quad(A \in \mathfrak{B}(\mathbb{R})),
$$

and hence we may assume always that $\mu$ is an even measure. Thus we let

$$
\mathbf{M}_{e}(\mathbb{R}):=\{\mu \in \mathbf{M}(\mathbb{R}) \mid \mu \text { is even }\}
$$

and note that this is a closed subalgebra of the convolution algebra $\mathbf{M}(\mathbb{R})$ of all bounded measures on $\mathbb{R}$.

Proposition 2.1 Let $(\operatorname{Cos}(s))_{s \in \mathbb{R}}$ be a bounded cosine function. Then the mapping

$$
\left(\mu \longmapsto T_{\mu}\right): \mathbf{M}_{e}(\mathbb{R}) \longrightarrow \mathcal{L}(X)
$$

is a bounded homomorphism of Banach algebras.

Proof Let $\mu, v \in \mathbf{M}_{e}(\mathbb{R})$ and $x \in X$. We compute

$$
\begin{aligned}
T_{\mu} T_{\nu} x & =\int_{\mathbb{R}} \int_{\mathbb{R}} \operatorname{Cos}(t) \operatorname{Cos}(s) x v(\mathrm{~d} s) \mu(\mathrm{d} t) \\
& =\frac{1}{2} \int_{\mathbb{R}} \int_{\mathbb{R}} \operatorname{Cos}(t+s) x v(\mathrm{~d} s) \mu(\mathrm{d} t)+\frac{1}{2} \int_{\mathbb{R}} \int_{\mathbb{R}} \operatorname{Cos}(t-s) x v(\mathrm{~d} s) \mu(\mathrm{d} t) \\
& \stackrel{(*)}{=} \int_{\mathbb{R}} \int_{\mathbb{R}} \operatorname{Cos}(t+s) x v(\mathrm{~d} s) \mu(\mathrm{d} t) \\
& =\int_{\mathbb{R}} \operatorname{Cos}(s) x(\mu * v)(\mathrm{d} s)=T_{\mu * v} x .
\end{aligned}
$$


In equality $(*)$ we performed a change of variable $s \mapsto-s$ in the second integral and used that $v$ is an even measure. That $\left\|T_{\mu}\right\| \leq \sup _{s \in \mathbb{R}}\|\operatorname{Cos}(s)\|\|\mu\|_{\mathbf{M}}$ is obvious.

For $\mu \in \mathbf{M}(\mathbb{R})$ we define its cosine transform by $\mathcal{C} \mu:=\mathcal{F}\left(\mu_{e}\right)$, i.e.,

$$
(\mathcal{C} \mu)(t)=\int_{\mathbb{R}} \cos (s t) \mu(\mathrm{d} s) \quad(t \in \mathbb{R}) .
$$

Evidently, if $\mu$ is even then $\mathcal{C} \mu$ coincides with the Fourier transform of $\mu$. Moreover, $\mathcal{C} \mu$ is always an even function, hence is determined by its restriction to $\mathbb{R}_{+}$. Therefore often we shall not distinguish between a function defined on $\mathbb{R}_{+}$and its even extension to $\mathbb{R}$. Let

$$
\mathcal{E}\left(\mathbb{R}_{+}\right):=\{\mathcal{C} \mu \mid \mu \in \mathbf{M}(\mathbb{R})\}=\left\{\mathcal{F}(\mu) \mid \mu \in \mathbf{M}_{e}(\mathbb{R})\right\} .
$$

Then $\mathcal{E}\left(\mathbb{R}_{+}\right)$is an algebra with respect to pointwise multiplication of functions. If $f=\mathcal{C} \mu \in$ $\mathcal{E}\left(\mathbb{R}_{+}\right)$we define $\Phi(f):=T_{\mu}$, which is a good definition since the Fourier transform is injective and $T_{\mu}=T_{\mu_{e}}$. The mapping

$$
\Phi: \mathcal{E}\left(\mathbb{R}_{+}\right) \longrightarrow \mathcal{L}(X)
$$

is a homomorphism of algebras, by Proposition 2.1 and the product law of the Fourier transform. Note that for $\lambda>0$ and $\mu:=(1 / 2) e^{-\lambda|s|} \mathrm{d} s$ we have

$$
f(t):=\mathcal{C}(\mu)(t)=\widehat{\mu}(t)=\frac{\lambda}{\lambda^{2}+t^{2}} \quad(t \in \mathbb{R})
$$

and

$$
\Phi(f)=T_{\mu}=\int_{0}^{\infty} e^{-\lambda s} \operatorname{Cos}(s) \mathrm{d} s=\lambda R\left(\lambda^{2},-A\right)=\lambda\left(\lambda^{2}+A\right)^{-1} .
$$

This is clearly an injective operator. Hence $(\mathcal{E}, \Phi)$ is a proper primary functional calculus, and we may choose freely any superalgebra $\mathcal{F}$ of $\mathcal{E}$ to obtain a proper abstract functional calculus in the sense of [10, Chap. 1]. We might take for example the algebra of all functions from $\mathbb{R}_{+}$to $\mathbb{C}$. A function $f: \mathbb{R}_{+} \longrightarrow \mathbb{C}$ is called regularizable if there is $e \in \mathcal{E}\left(\mathbb{R}_{+}\right)$such that $e f \in \mathcal{E}\left(\mathbb{R}_{+}\right)$as well and $\Phi(e)$ is injective. In this case, $e$ is called a regularizer for $f$, and the (closed but potentially unbounded) operator $\Phi(f)$ is defined as

$$
\Phi(f):=\Phi(e)^{-1} \Phi(e f) .
$$

This definition does not depend on the chosen regularizer and is also compatible with the definition of $\Phi$ on the algebra $\mathcal{E}\left(\mathbb{R}_{+}\right)$; moreover, it obeys the standard rules for unbounded functional calculi. See [10, Chap. 1] for proofs of these facts and more information.

To identify regularizable functions, we recall the well-known Bernstein lemma.

Lemma 2.2 Let $f \in H^{1}(\mathbb{R})$, i.e., $f, f^{\prime} \in \mathbf{L}^{\mathbf{2}}(\mathbb{R})$. Then $f \in \mathcal{F}\left(\mathbf{L}^{\mathbf{1}}(\mathbb{R})\right)$. Moreover, the mapping $\mathcal{F}^{-1}: H^{1}(\mathbb{R}) \longrightarrow \mathbf{L}^{\mathbf{1}}(\mathbb{R})$ is continuous.

Proof See [1, Lemma 8.2.1].

Here is a first application.

Lemma 2.3 Let $f \in \mathbf{C}^{\mathbf{1}}\left(\mathbb{R}_{+}\right)$such that $f^{\prime}$ is polynomially bounded. Then $f$ is regularizable, whence $\Phi(f)$ is defined. 
Proof As usual we view $f$ as an even function on $\mathbb{R}$. The hypotheses imply that $f$ is polynomially bounded. Let $g(t):=f(t)\left(1+t^{2}\right)^{-n}$ for $n \in \mathbb{N}$ large enough such that $g \in \mathbf{L}^{\mathbf{2}}(\mathbb{R})$ and $f^{\prime}(t)\left(1+t^{2}\right)^{-n} \in \mathbf{L}^{2}(\mathbb{R})$. Then

$$
g^{\prime}(t)=\frac{\left(1+t^{2}\right)^{n} f^{\prime}(t)-2 n t f(t)\left(1+t^{2}\right)^{n-1}}{(1+t)^{2 n}}=\frac{f^{\prime}(t)}{\left(1+t^{2}\right)^{n}}-\frac{2 n t}{\left(1+t^{2}\right)} g(t)
$$

for $t \neq 0$. Hence $g^{\prime} \in \mathbf{L}^{\mathbf{2}}(\mathbb{R})$ and by Bernstein's lemma it follows that $g \in \mathcal{F}\left(\mathbf{L}^{\mathbf{1}}(\mathbb{R})\right)$. As $g$ is even, $g \in \mathcal{E}\left(\mathbb{R}_{+}\right)$and since $\Phi\left(\left(1+t^{2}\right)^{-n}\right)=(1+A)^{-n}$ is injective, $f$ is regularizable.

Using this lemma one sees that the function $f(t)=|t|$ is regularizable by the function $\left(1+t^{2}\right)^{-1}$, and hence the operator

$$
B:=\Phi(f)=\Phi(|t|)
$$

is defined. From the definition it is immediate that $\mathcal{D}(A) \subset \mathcal{D}(B)$, and so $B$ is densely defined. Moreover, for $\lambda \notin \mathbb{R}_{+}$, the function $(\lambda-|t|)^{-1}$ is in $H^{1}(\mathbb{R})$, whence in $\mathcal{E}\left(\mathbb{R}_{+}\right)$. Therefore

$$
(\lambda-B)^{-1}=\Phi\left(\frac{1}{\lambda-|t|}\right) \in \mathcal{L}(X) .
$$

This shows that $\sigma(B) \subset \mathbb{R}_{+}$.

It is reasonable to say that the functional calculus $\Phi$ is a functional calculus for $B$ and write $f(B)$ instead of $\Phi(f)$. In Sect. 6 we shall show that $B=A^{1 / 2}$, but that is unimportant at the moment. We note that also the functions $f_{s}, s \in \mathbb{R}$, defined by

$$
f_{S}(t):=e^{-i s|t|} \quad(t \in \mathbb{R})
$$

satisfy the conditions of the lemma. Hence we obtain the operators

$$
U(s):=\Phi\left(e^{-i s|t|}\right)=\left[e^{-i s|t|}\right](B) \quad(s \in \mathbb{R}) .
$$

Our main goal is to show that if $X$ is a UMD space, then $U$ is a bounded $C_{0}$-group on $X$. Functional calculus then yields that indeed

$$
\operatorname{Cos}(s)=[\cos (t)](B)=\left(\frac{e^{-i s|t|}+e^{i s|t|}}{2}\right)(B)=\frac{U(s)+U(-s)}{2} \quad(s \in \mathbb{R})
$$

and the first step in the proof of Theorem 1.1 is complete.

Lemma 2.4 If $U(s)=\left[e^{-i s|t|}\right](B)$ is a bounded operator for every $s \in \mathbb{R}$, then $(U(s))_{s \in \mathbb{R}}$ is a $C_{0}$-group and its generator is $-i B$.

Proof Suppose that the hypothesis of the lemma holds true. General functional calculus theory yields that $U$ is a group. To prove that $U$ is strongly continuous by classical semigroup theory [12, Theorem 10.2.3] it suffices to show that the trajectories $U(\cdot) x, x \in X$, are all measurable. Since $\mathcal{D}(A)$ is dense in $X$, it suffices to show that $U(\cdot) x$ is continuous for each $x \in \mathcal{D}(A)$. Hence we consider the functions

$$
g_{s}(t):=\frac{e^{-i s|t|}}{1+t^{2}} \quad(t, s \in \mathbb{R})
$$

and by Bernstein's lemma it suffices to show that $\left(s \longmapsto g_{s}\right): \mathbb{R} \longrightarrow H^{1}(\mathbb{R})$ is continuous. This is easy to see. 
Now, let $-C$ be the generator of $U$. To prove that $i B=C$, note that $\left\|g_{s}\right\|_{H^{1}}=O(|s|)$ as $|s| \rightarrow \infty$. Hence one can take Laplace transforms within $H^{1}(\mathbb{R})$ and obtains for large $\lambda>0$

$$
\begin{aligned}
(1+A)^{-1}(\lambda+C)^{-1} & =\int_{0}^{\infty} e^{-\lambda s} U(s)(1+A)^{-1} \mathrm{~d} s=\int_{0}^{\infty} e^{-\lambda s} \Phi\left(g_{s}\right) \mathrm{d} s \\
& =\Phi\left(\int_{0}^{\infty} e^{-\lambda s} g_{s} \mathrm{~d} s\right)=\Phi\left(\frac{1}{\left(1+t^{2}\right)(\lambda+i|t|)}\right) \\
& =(1+A)^{-1}(\lambda+i B)^{-1}
\end{aligned}
$$

This shows that $C=i B$.

Finally, we state a "convergence lemma" for our functional calculus.

Lemma 2.5 Let $\left(f_{\alpha}\right)_{\alpha}$ be a net of continuous functions on $\mathbb{R}_{+}$converging pointwise to a function $f$ and satisfying the following conditions:

(1) $f_{\alpha} /\left(1+t^{2}\right) \in H^{1}(\mathbb{R})$ for all $\alpha$.

(2) $f_{\alpha} /\left(1+t^{2}\right) \rightarrow f /\left(1+t^{2}\right)$ within $H^{1}(\mathbb{R})$.

Then $f_{\alpha}(B) x \rightarrow f(B) x$ for all $x \in \mathcal{D}(A)$. If in addition

(3) $\sup _{\alpha}\left\|f_{\alpha}(B)\right\|<\infty$,

then $f(B) \in \mathcal{L}(X)$ and $f_{\alpha}(B) \rightarrow f(B)$ strongly.

Proof Since $\mathcal{D}(A)$ is dense in $X$, it suffices to show that

$$
f_{\alpha}(B)(1+A)^{-1} \rightarrow f(B)(1+A)^{-1}
$$

in norm. This is guaranteed by conditions (1) and (2) and Bernstein's lemma.

Note that the hypotheses of Lemma 2.5 imply that $f_{\alpha} \rightarrow f$ uniformly on compact subsets of $\mathbb{R}_{+}$. On the other hand, the hypotheses (1) and (2) of Lemma 2.5 are clearly satisfied if one has the following situation:

(1) $f \in \mathbf{C}^{\mathbf{1}}\left(\mathbb{R}_{+}\right)$and $f_{\alpha} \in \mathbf{C}^{\mathbf{1}}\left(\mathbb{R}_{+}\right)$for all $\alpha$;

(2) $f_{\alpha} \rightarrow f$ and $f_{\alpha}^{\prime} \rightarrow f^{\prime}$ uniformly on compact subsets of $\mathbb{R}_{+}$;

(3) $\sup _{\alpha}\left\|f_{\alpha}\right\|_{\infty}+\left\|f_{\alpha}^{\prime}\right\|_{\infty}<\infty$.

An example for this situation is given, for fixed $s \in \mathbb{R}$, by the functions

$$
f_{\alpha}(t):=e^{-(\alpha+i s)|t|}, \quad f(t):=e^{-i s|t|} \quad(t \in \mathbb{R}, 0<\alpha \leq 1)
$$

viewed as a net for $\alpha \searrow 0$. Let us define

$$
T_{B}(\lambda):=\Phi\left(e^{-\lambda|t|}\right)=\left[e^{-\lambda|t|}\right](B) \quad(\operatorname{Re} \lambda>0) .
$$

The following proposition shows that things behave as expected.

Proposition 2.6 Let $U$ and $T_{B}$ be defined as above. Then the following assertions hold.

(a) $T_{B}(\lambda) x=\frac{\lambda}{\pi} \int_{0}^{\infty} \frac{\operatorname{Cos}(s) x}{\lambda^{2}+s^{2}} \mathrm{~d} s \quad(x \in X, \operatorname{Re} \lambda>0)$. 
(b) $T_{B}:\{\operatorname{Re} \lambda>0\} \longrightarrow \mathcal{L}(X)$ is holomorphic and satisfies the semigroup law, and for each $\varphi \in(0, \pi / 2)$ one has

$$
\sup \{\|T(\lambda)\||\lambda \neq 0,| \arg \lambda \mid \leq \varphi\}<\infty
$$

(c) $-B$ is the generator of the semigroup $T_{B}$.

(d) For each $s \in \mathbb{R}, U(s)$ is a bounded operator if and only if

$$
\sup _{0<\alpha \leq 1}\left\|T_{B}(\alpha+i s)\right\|<\infty,
$$

and in this case $U(s) x=\lim _{\alpha \searrow 0} T_{B}(\alpha+i s) x \quad(x \in X)$.

Property (b) can be abbreviated by saying that $T_{B}$ is a bounded holomorphic semigroup of angle $\pi / 2$, see [1, Definition 7.3.7].

Proof (a) follows from the fact that

$$
\mathcal{F}^{-1}\left(e^{-\lambda|t|}\right)(s)=\frac{\lambda}{\pi\left(\lambda^{2}+s^{2}\right)}=: g_{\lambda}(s) \quad(s \in \mathbb{R}) .
$$

(b) The semigroup law is just the multiplicativity of the functional calculus. Holomorphy follows since the mapping

$$
\left(\lambda \longmapsto g_{\lambda}\right):\{\operatorname{Re} \lambda>0\} \longrightarrow \mathbf{L}^{\mathbf{1}}(\mathbb{R})
$$

is holomorphic and the remaining statement follows from the identity

$$
\left\|g_{\lambda}\right\|_{\mathbf{L}^{1}}=\frac{2}{\pi} \int_{0}^{\infty} \frac{1}{\left|(\lambda /|\lambda|)^{2}+s^{2}\right|} \mathrm{d} s \quad(\operatorname{Re} \lambda>0) .
$$

(c) Note that $\left\|g_{r}\right\|_{\mathbf{L}^{\mathbf{1}}}=1$ for all $r>0$. Hence one may take Laplace transforms in $\mathbf{L}^{\mathbf{1}}$ and this shows that

$$
\int_{0}^{\infty} e^{-z r} T_{B}(r) d r=\Phi\left(\int_{0}^{\infty} e^{-z r} e^{-r t} \mathrm{~d} r\right)=\Phi\left(\frac{1}{z+t}\right)=(z+B)^{-1}
$$

for $z>0$.

(d) By abstract functional calculus we have

$$
T_{B}(\alpha+i s)=\left[e^{-\alpha|t|} e^{-i s|t|}\right](B)=U(s) T_{B}(\alpha)
$$

Since $T_{B}$ is a bounded semigroup, if $U(s)$ is bounded then the operators $T_{B}(\alpha+i s), \alpha>0$, are uniformly bounded. Conversely, supposing that these operators are uniformly bounded one can apply the convergence lemma (Lemma 2.5) and the remarks following it.

As a consequence we note that to prove the first part of Theorem 1.1 we only have to establish the uniform boundedness

$$
\sup \left\{\left\|T_{B}(\lambda)\right\| \mid \operatorname{Re} \lambda>0\right\}<\infty .
$$

This will be done with the help of a transference principle, which is the topic of the following section. 
Remark 2.7 The idea to reduce the proof of Theorem 1.1 to the uniform boundedness of the operator family $\left(T_{B}(\lambda)\right)_{\operatorname{Re} \lambda>0}$ is taken from the paper by Cioranescu and Keyantuo [5]. These authors employ the theory of boundary values of holomorphic semigroups as it is presented in [1, Sect. 3.9]. That theory can be incorporated into the general theory of functional calculus, but doing so here would certainly take us too far astray. We decided to give an ad hoc proof based on functional calculus methods in order to keep the presentation as self-contained as possible and to demonstrate once more the power of functional calculus theory.

Let us also mention that the more recent paper [14] treats the Phillips calculus in a wider context under the name "vector-valued cosine transform" and uses it to characterize general cosine functions on Banach spaces.

\section{The transference result}

Let us begin with some abstract considerations. Suppose one is given an operator $T$ on a Banach space $X$ and wants to estimate its norm. Transference means that one factorises the "bad" operator $T$ over a second Banach space $Y$ via mappings $\iota: X \longrightarrow Y, S: Y \longrightarrow Y$ and $P: Y \longrightarrow X$, i.e., $T=P S \iota$. This means that the diagram

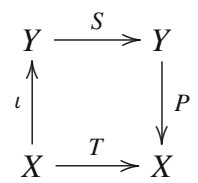

commutes. The operator $S$ is hopefully "better" than $T$ in the sense that one has reasonable estimates on its norm. The factorization leads to estimates of the form $\|T\| \leq c\|S\|$. It is possible in certain cases to keep $S$ fixed while varying $\iota, P$, thereby improving on $c$.

A classical example is the transference principle by Coifman and Weiss [6,7] in its abstract form given by Berkson et al. [2]. It has the form

$$
\left\|\int_{\mathbb{R}} U(s) x \mu(\mathrm{d} s)\right\| \leq M^{2}\left\|L_{\mu}\right\|_{\mathcal{L}\left(\mathbf{L}^{\boldsymbol{p}}(\mathbb{R} ; X)\right)} \quad(\mu \in \mathbf{M}(\mathbb{R})),
$$

where $U$ is a bounded $C_{0}$-group on a Banach space $X, M:=\sup _{s \in \mathbb{R}}\|U(s)\|$ is its bound, and $L_{\mu}$ denotes the convolution operator

$$
L_{\mu}:=(f \longmapsto \mu * f)
$$

on each space where it is meaningful. Such an estimate is particularly useful if the Banach space is a UMD space, because then one can use Fourier multiplier theory to estimate the norm of $L_{\mu}$. We aim at the analogous result when the group $U$ is replaced by a cosine function.

Theorem 3.1 Let $(\operatorname{Cos}(t))_{t \in \mathbb{R}}$ be a bounded cosine function on a Banach space $X$, and let $T_{\mu}$ be defined by

$$
T_{\mu} x=\int_{\mathbb{R}} \operatorname{Cos}(s) x \mu(\mathrm{d} s) \quad\left(x \in X, \mu \in \mathbf{M}_{e}(\mathbb{R})\right) .
$$


Then

$$
\left\|T_{\mu}\right\| \leq 5 M^{2}\left\|L_{\mu}\right\|_{\mathcal{L}\left(\mathbf{L}^{p}(\mathbb{R} ; X)\right)}\left(\mu \in \mathbf{M}_{e}(\mathbb{R})\right),
$$

where $M:=\sup _{s \in \mathbb{R}}\|\operatorname{Cos}(s)\|$ and $p \in[1, \infty)$.

Proof Fix $p \in[1, \infty)$ and suppose first that $\mu$ has support within the interval $[-N, N]$. We want to factorise $T_{\mu}$ over $Y:=\mathbf{L}^{p}(\mathbb{R} ; X)$, with $S=L_{\mu}$ being the convolution with $\mu$. Since $\mu$ is an even measure, one has for $x \in X$ and $|t| \leq n$

$$
\begin{aligned}
\operatorname{Cos}(t) T_{\mu} x & =\int_{-N}^{N} \operatorname{Cos}(t) \operatorname{Cos}(s) x \mu(\mathrm{d} s) \\
& =\frac{1}{2}\left[\int_{-N}^{N} \operatorname{Cos}(t-s) x \mu(\mathrm{d} s)+\int_{-N}^{N} \operatorname{Cos}(t+s) x \mu(\mathrm{d} s)\right] \\
& =\int_{-N}^{N} \operatorname{Cos}(t-s) x \mu(\mathrm{d} s)=\left[\mu *\left(\iota_{n} x\right)\right](t)=\left(L_{\mu} \iota_{n} x\right)(t),
\end{aligned}
$$

where $\iota_{n}: X \longrightarrow Y$ is given by

$$
\iota_{n}(x)=\left[s \longmapsto \mathbf{1}_{[-(N+n),(N+n)]}(s) \operatorname{Cos}(s) x\right] \in \mathbf{L}^{p}(\mathbb{R} ; X) \quad(x \in X) .
$$

To determine $P_{n}: Y \longrightarrow X$ such that $T_{\mu}=P_{n} L_{\mu} \iota_{n}$, note that by the defining identity for cosine functions (1.1) one has $x=2 \operatorname{Cos}(t)^{2} x-\operatorname{Cos}(2 t) x$ and hence

$$
x=\frac{1}{n} \int_{-n / 2}^{n / 2} x \mathrm{~d} t=\frac{2}{n} \int_{-n / 2}^{n / 2} \operatorname{Cos}(t)^{2} x \mathrm{~d} t-\frac{1}{2 n} \int_{-n}^{n} \operatorname{Cos}(t) x \mathrm{~d} t \quad(x \in X) .
$$

If we replace $x$ by $T_{\mu} x$ in this identity and recall that $\operatorname{Cos}(t) T_{\mu} x=\left(L_{\mu} \iota_{n} x\right)(t)$ for $|t| \leq n$, we see that

$$
T_{\mu}=P_{n} L_{\mu} \iota_{n},
$$

where $P_{n}: Y \longrightarrow X$ is defined by

$$
P_{n} f:=\frac{2}{n} \int_{-n / 2}^{n / 2} \operatorname{Cos}(t) f(t) \mathrm{d} t-\frac{1}{2 n} \int_{-n}^{n} f(t) \mathrm{d} t \quad(f \in Y) .
$$

Let us estimate norms. One clearly has

$$
\left\|\iota_{n} x\right\|_{\mathbf{L}^{p}}=\left(\int_{-(n+N)}^{n+N}\|\operatorname{Cos}(s) x\|^{p} \mathrm{~d} s\right)^{\frac{1}{p}} \leq(2 n+2 N)^{\frac{1}{p}} M\|x\| \quad(x \in X),
$$


and hence $\left\|\iota_{n}\right\| \leq(2 n+2 N)^{\frac{1}{p}} M$. On the other hand, for $f \in Y=\mathbf{L}^{\boldsymbol{p}}(\mathbb{R} ; X)$

$$
\begin{aligned}
\left\|P_{n} f\right\| & \leq \frac{2 M}{n} \int_{-n / 2}^{n / 2}\|f(t)\| \mathrm{d} t+\frac{1}{2 n} \int_{-n}^{n}\|f(t)\| \mathrm{d} t \\
& \leq \frac{4 M+1}{2 n} \int_{-n}^{n}\|f(t)\| \mathrm{d} t \leq \frac{4 M+1}{2 n}(2 n)^{1 / p^{\prime}}\|f\|_{\mathbf{L}^{p}([-n, n] ; X)} \\
& \leq 5 M(2 n)^{-1 / p}\|f\|_{Y}
\end{aligned}
$$

by Hölder's inequality. Hence $\left\|P_{n}\right\| \leq 5 M(2 n)^{-1 / p}$. Combining these estimates yields

$$
\left\|T_{\mu}\right\| \leq 5 M^{2}\left(1+\frac{N}{n}\right)^{\frac{1}{p}}\left\|L_{\mu}\right\|_{\mathcal{L}(Y)} .
$$

But $n$ was arbitrary, and so we can let $n \rightarrow \infty$ to obtain

$$
\left\|T_{\mu}\right\| \leq 5 M^{2}\left\|L_{\mu}\right\|_{\mathcal{L}\left(\mathbf{L}^{p}(\mathbb{R} ; X)\right)} .
$$

As a last step we remove the support restriction on $\mu$. For a general even measure $\mu$ the sequence of measures $\mu_{n}(\mathrm{~d} s):=\mathbf{1}_{[-n, n]}(s) \mu(\mathrm{d} s)$ converges to $\mu$ in the total variation norm. This implies convergence $L_{\mu_{n}} \rightarrow L_{\mu}$ in $\mathcal{L}\left(\mathbf{L}^{p}(\mathbb{R} ; X)\right)$ by Young's inequality, and $T_{\mu_{n}} \rightarrow T_{\mu}$ in $\mathcal{L}(X)$. The theorem is completely proved.

To make effective use of Theorem 3.1, one has to have good estimates for the norm of the convolution operators $L_{\mu}$. This is the topic of the next section.

\section{UMD spaces and proof of main theorem}

Let us recall the notion of a bounded Fourier multiplier. Fix $p \in[1, \infty)$. A function $m \in$ $\mathbf{L}^{\infty}(\mathbb{R})$ is called a bounded $\mathbf{L}^{p}(\mathbb{R} ; X)$-Fourier multiplier if there is a constant $c=c(m, p, X)$ such that

$$
\left\|\mathcal{F}^{-1}(m \widehat{f})\right\|_{\mathbf{L}^{p}(\mathbb{R} ; X)} \leq c\|f\|_{\mathbf{L}^{p}(\mathbb{R} ; X)}
$$

for all functions $f$ belonging to the Schwartz class $\mathcal{S}(\mathbb{R} ; X)$. In this case the operator $\mathcal{T}_{m}$ given by

$$
\mathcal{T}_{m} f:=\mathcal{F}^{-1}(m \widehat{f}) \quad(f \in \mathcal{S}(\mathbb{R} ; X))
$$

extends to a bounded operator on $\mathbf{L}^{\boldsymbol{p}}(\mathbb{R} ; X)$, and the function $m$ is called the symbol of $\mathcal{T}_{m}$. We set

$$
\mathcal{M}_{p}(\mathbb{R} ; X):=\left\{m \in \mathbf{L}^{\infty}(\mathbb{R}) \mid m \text { is a bounded } \mathbf{L}^{p}(\mathbb{R} ; X) \text {-Fourier multiplier }\right\}
$$

with the norm $\|m\|_{\mathcal{M}_{p}(\mathbb{R} ; X)}=\left\|\mathcal{T}_{m}\right\|_{\mathcal{L}\left(\mathbf{L}^{p}(\mathbb{R} ; X)\right)}$. The following lemma collects some useful facts.

Lemma 4.1 Let $p \in[1, \infty)$ and let $X$ be a Banach space. Then the following assertions hold.

(a) If $\mu \in \mathbf{M}(\mathbb{R})$ then $\widehat{\mu} \in \mathcal{M}_{p}(\mathbb{R} ; X)$ and $\mathcal{T}_{\widehat{\mu}}=L_{\mu}$ is convolution with $\mu$.

(b) The space $\mathcal{M}_{p}(\mathbb{R} ; X)$ is a Banach algebra. 
(c) If $m \in \mathcal{M}_{p}(\mathbb{R} ; X)$ then for $\alpha, \beta, \gamma \in \mathbb{R}, \beta \neq 0$,

$$
m_{\alpha, \beta, \gamma}(t):=\mathrm{e}^{-i \alpha t} m(\beta t+\gamma) \in \mathcal{M}_{p}(\mathbb{R} ; X)
$$

with $\left\|m_{\alpha, \beta, \gamma}\right\|_{\mathcal{M}_{p}(\mathbb{R} ; X)}=\|m\|_{\mathcal{M}_{p}(\mathbb{R} ; X)}$.

(d) $\mathcal{M}_{1}(\mathbb{R} ; X)=\mathcal{F} \mathbf{M}(\mathbb{R})$.

(e) If $X=H$ is a Hilbert space then $\mathcal{M}_{2}(\mathbb{R} ; H)=\mathbf{L}^{\infty}(\mathbb{R})$ with

$$
\|m\|_{\mathcal{M}_{2}(\mathbb{R} ; H)}=\|m\|_{\infty} .
$$

Proof These facts are standard and can be found in many books.

A Banach space $X$ is called HT-space if the function

$$
h(t):=-i \operatorname{sgn} t \quad(t \in \mathbb{R})
$$

is a bounded $\mathbf{L}^{\mathbf{2}}(\mathbb{R} ; X)$-multiplier. The associated operator $\mathcal{H}:=\mathcal{T}_{h}$ is called the Hilbert transform. It is well known that one may replace $\mathbf{L}^{2}$ by any $\mathbf{L}^{p}, p \in(1, \infty)$ in this definition. Moreover, if $X$ is a HT-space then

$$
\mathcal{H} f(t)=\lim _{\varepsilon \rightarrow 0} \int_{\varepsilon \leq|s| \leq 1 / \varepsilon} \frac{f(t-s)}{s} \mathrm{~d} s
$$

in the $\mathbf{L}^{\boldsymbol{p}}(\mathbb{R} ; X)$-sense. (Actually, one can assert also convergence pointwise almost everywhere, but this is of no importance in this paper.) After work of Burkholder [4] and Bourgain [3], the HT-property can be equivalently characterised by the so-called UMD-property, involving $u$ nconditional martingale differences. We shall not make use of this characterization, but nevertheless use the name "UMD space", as this is now common.

Suppose that $-A$ generates a bounded cosine function $(\operatorname{Cos}(s))_{s \in \mathbb{R}}$ on a UMD space $X$. The semigroup generated by $-B$ (which actually is the same as $-A^{1 / 2}$, see Sect. 6) is given by the Phillips calculus:

$$
T_{B}(\lambda)=T_{\mu_{\lambda}} \text { with } \widehat{\mu_{\lambda}}(t)=\mathrm{e}^{-\lambda|t|} \quad(t \in \mathbb{R}) .
$$

By the transference principle (Theorem 3.1) one has

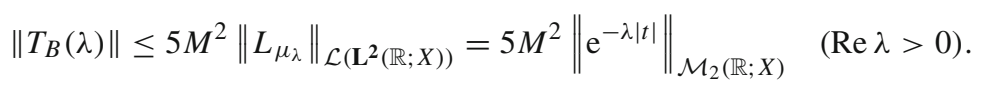

Now, writing $\lambda=\alpha+i s$ with $\alpha>0, s \in \mathbb{R}$ :

$$
\mathrm{e}^{-\lambda|t|}=\mathrm{e}^{-\alpha|t|}(\cos (s t)+h(t) \sin (s t)) \quad(t \in \mathbb{R})
$$

as a simple computation shows. The symbols $\mathrm{e}^{-|t|}, \sin (t), \cos (t)$ are all in $\mathcal{F} \mathbf{M}(\mathbb{R})$, hence in $\mathcal{M}_{2}(\mathbb{R} ; X)$, and also $h \in \mathcal{M}_{2}(\mathbb{R} ; X)$, since $X$ is a UMD space. Thus it follows from Lemma 4.1 that the family $\left(\mathrm{e}^{-\lambda|t|}\right)_{\operatorname{Re} \lambda>0}$ is uniformly bounded in $\mathcal{M}_{2}(\mathbb{R} ; X)$. Hence we have proved the following statement.

Proposition 4.2 Let - A generate a bounded cosine function $(\operatorname{Cos}(s))_{s \in \mathbb{R}}$ on the UMD space $X$. Then the holomorphic semigroup $\left(T_{B}(\lambda)\right)_{\operatorname{Re} \lambda>0}$ is uniformly bounded.

As was explained in Sect. 2, this completes the proof of the first part of Theorem 1.1. In the next section we shall provide a second approach to this result. 


\section{An alternative approach}

If $\operatorname{Cos}: \mathbb{R} \longrightarrow \mathcal{L}(X)$ is a cosine function, the associated sine function $\operatorname{Sin}: \mathbb{R} \longrightarrow \mathcal{L}(X)$ is defined by

$$
\operatorname{Sin}(s) x:=\int_{0}^{s} \operatorname{Cos}(r) x \mathrm{~d} r \quad(x \in X, s \in \mathbb{R}),
$$

see [1, p. 210]. In this section we shall give a new (and simpler) proof of a representation formula for the (in general unbounded) operators $A^{1 / 2} \operatorname{Sin}(s), s \in \mathbb{R}$, originally due to Fatttorini. As we shall see shortly, the reduction group is given by

$$
U(s)=\operatorname{Cos}(s)-i A^{1 / 2} \operatorname{Sin}(s) \quad(s \in \mathbb{R}),
$$

and this will lead to an alternative proof of Theorem 1.1.

The approach uses the standard formula

$$
\mathrm{e}^{-i s|t|}=\cos (s t)-i \operatorname{sgn}(t) \sin (s t) \quad(s, t \in \mathbb{R}) .
$$

Inserting $B$ (which below will be proved to be equal to $A^{1 / 2}$ ) yields

$$
U(s)=\mathrm{e}^{-i s B}=\operatorname{Cos}(s)-i B \operatorname{Sin}(s) \quad(s \in \mathbb{R})
$$

since, for $s \in \mathbb{R}$,

$$
\left(\frac{\sin (s|t|)}{|t|}\right)(B)=\left(\int_{0}^{s} \cos (r|t|) \mathrm{d} r\right)(B)=\int_{0}^{s} \operatorname{Cos}(r) \mathrm{d} r=\operatorname{Sin}(s) .
$$

(One has indeed equality in (5.1), by general functional calculus [10, Theorem 1.3.2] and since $\operatorname{Cos}(s)$ is a bounded operator).

Theorem 5.1 Let - A generate a bounded cosine function Cos on a Banach space $X$, and let $S(s):=[\operatorname{sgn}(t) \sin (s t)](B)=B \operatorname{Sin}(s), s \in \mathbb{R}$. Then

$$
S(s) x=\frac{1}{\pi} \mathrm{PV}-\int_{\mathbb{R}} \frac{\operatorname{Cos}(s-r) x}{r} \mathrm{~d} r \quad(s \in \mathbb{R}) .
$$

for all $x \in \mathcal{D}(A)$. In the case that $X$ is a UMD space, $\sup _{s \in \mathbb{R}}\|S(s)\|<\infty$, and the representation (5.2) holds for all $x \in X$.

The formula (5.2) is due to Fattorini [8]. The extension in the UMD case was established by [5, Proof of Theorem 2.5]; in their proof the authors make use of Burkholder's result that in a UMD space the Hilbert transform converges almost everywhere. Moreover, some intricate measure-theoretic arguments are also needed. Our approach does not need more than the mere definition of UMD space, as well as some mild Fourier analysis and the functional calculus constructed in Sect. 2.

In the proof of Theorem 5.1 we shall have occasion to use the function

$$
H(t):=\int_{0}^{1} \frac{\sin (s t)}{s} \mathrm{~d} s \quad(t \in \mathbb{R})
$$


which is odd, bounded and has bounded derivative. A classical fact is the identity

$$
\lim _{t \rightarrow \pm \infty} H(t)=\lim _{t \rightarrow \pm \infty} \int_{0}^{t} \frac{\sin s}{s} \mathrm{~d} s=\frac{ \pm \pi}{2} .
$$

Let us also introduce the function

$$
F(t):=\operatorname{sgn}(t) H(t)-\frac{\pi}{2}=-\int_{|t|}^{\infty} \frac{\sin r}{r} \mathrm{~d} r \quad(t \in \mathbb{R})
$$

which is continuous, even and vanishes at infinity, and finally

$$
G_{s, c}(t):=\operatorname{sgn}(t) \sin (s t) F(c t) \quad(t \in \mathbb{R}, s \in \mathbb{R}, c>0) .
$$

Now look at the stated convergence of the principal value integral (5.2). A simple calculation yields

$$
\begin{aligned}
\int_{a \leq|r| \leq b} \frac{\cos [(s-r) t]}{r} \mathrm{~d} r & =2 \sin (s t) \int_{a}^{b} \frac{\sin (r t)}{r} \mathrm{~d} r \\
& =2 \sin (s t)[H(b t)-H(a t)]=2 \operatorname{sgn}(t) \sin (s t)[F(b t)-F(a t)] \\
& =2 G_{s, b}(t)-2 G_{s, a}(t)
\end{aligned}
$$

for $t \in \mathbb{R}$. We have to look what happens as $b$ tends to $\infty$ and $a$ tends to 0 .

Lemma 5.2 The following assertions hold.

(a) $F \in \mathcal{F}\left(\mathbf{L}^{\mathbf{1}}(\mathbb{R})\right)$.

(b) For all $s \in \mathbb{R}$ and $c>0$, the function $G_{s, c}$ is in $\mathcal{E}\left(\mathbb{R}_{+}\right)$.

(c) $G_{s, c} \rightarrow 0$ in $\mathcal{E}\left(\mathbb{R}_{+}\right)$as $c \rightarrow \infty$, in the sense that there exist measures $\mu_{s, c} \in \mathbf{M}(\mathbb{R})$ such that $\mathcal{C} \mu_{s, c}=G_{s, c}$ and $\mu_{s, c} \rightarrow 0$ in $\mathbf{M}(\mathbb{R})$.

(d) For fixed $s \in \mathbb{R}$

$$
\frac{G_{s, c}(t)}{1+t^{2}} \rightarrow \frac{-(\pi / 2) \operatorname{sgn}(t) \sin (s t)}{1+t^{2}} \text { as } c \searrow 0
$$

in $H^{1}(\mathbb{R})$ (as functions of $t$ ).

Proof (a) Since

$$
F(t)=-\int_{|t|}^{\infty} \frac{\sin s}{s} \mathrm{~d} s \quad(t \in \mathbb{R})
$$

we have $|t| F^{\prime}(t)=\sin (t)$, whence $F^{\prime} \in \mathbf{L}^{2}(\mathbb{R})$. For $t \neq 0$, integration by parts yields

$$
F(t)=-\int_{|t|}^{\infty} \frac{\sin r}{r} \mathrm{~d} r=\frac{\cos t}{|t|}+\frac{1}{|t|} \int_{1}^{\infty} \frac{\cos (t r)}{r^{2}} \mathrm{~d} r .
$$

This shows that also $|t| F(t)$ is bounded and hence $F \in \mathbf{L}^{\mathbf{2}}(\mathbb{R})$. We conclude that $F \in H^{1}(\mathbb{R})$ and therefore in $\mathcal{F}\left(\mathbf{L}^{\mathbf{1}}(\mathbb{R})\right)$, by Bernstein's Lemma 2.2. 
(b) and (c) Using the above integration by parts, we find

$$
G_{s, c}(t)=\left(\frac{\sin (s t)}{t}\right)\left(\frac{\cos (c t)}{c}+\int_{c}^{\infty} \frac{\cos (t r)}{r^{2}} \mathrm{~d} r\right) \quad(t \in \mathbb{R}) .
$$

Note that $\mathcal{C}\left(\mathbf{1}_{[0, s]}\right)=\sin (s t) / t$ and the second factor is the cosine transform of $\mu_{c}:=$ $c^{-1} \delta_{c}+\mathbf{1}_{[c, \infty)} r^{-2} \mathrm{~d} r$. This proves (b), and (c) follows readily since obviously $\mu_{c} \rightarrow 0$ in $\mathbf{M}(\mathbb{R})$ as $c \rightarrow \infty$.

(d) It is easily seen that $\operatorname{sgn}(t) \sin (s t)\left(1+t^{2}\right)^{-1} \in H^{1}(\mathbb{R})$. Furthermore,

$$
G_{s, c}(t)+(\pi / 2) \operatorname{sgn}(t) \sin (s t)=\sin (s t) H(c t)
$$

and it is likewise easy to see that

$$
\frac{\sin (s t) H(c t)}{1+t^{2}} \rightarrow 0 \quad(c \searrow 0)
$$

in $H^{1}(\mathbb{R})$, as functions of $t \in \mathbb{R}$. This completes the proof.

Returning to our starting point, we may insert the operator $B$ by means of the functional calculus and obtain (for fixed $s \in \mathbb{R}$ and $0<a<b<\infty$ )

$$
\frac{1}{\pi} \int_{a \leq|r| \leq b} \frac{\operatorname{Cos}(s-r)}{r} \mathrm{~d} r=\frac{2}{\pi}\left(G_{s, b}(B)-G_{s, a}(B)\right) .
$$

By (c) of Lemma 5.2 one has $\lim _{b \rightarrow \infty} G_{s, b}(B)=0$ in norm, and by (d) of Lemma 5.2 together with the convergence lemma (Lemma 2.5) one has

$$
-\frac{2}{\pi} G_{s, a}(B) x \rightarrow(\operatorname{sgn}(t) \sin (s t))(B) x=S(s) x \quad(a \searrow 0)
$$

for all $x \in \mathcal{D}(A)$. Moreover, the convergence is true for all $x \in X$ in the case that $\sup _{0<a \leq 1}\left\|G_{s, a}(B)\right\|<\infty$.

To complete the proof of Theorem 1.1 suppose that $X$ is a UMD space. It suffices to establish the uniform boundedness

$$
\sup _{0<a<b}\left\|\int_{a \leq|r| \leq b} \frac{\operatorname{Cos}(s-r)}{r} \mathrm{~d} r\right\|<\infty
$$

or, equivalently,

$$
\sup \left\{\left\|G_{s, c}(B)\right\| \mid s \in \mathbb{R}, c>0\right\}<\infty .
$$

By the transference principle (Theorem 3.1) and (5.3) it is sufficient to show that the family of $\mathbf{L}^{2}(\mathbb{R} ; X)$-Fourier multiplier operators with symbols

$$
\operatorname{sgn}(\cdot) \sin (s \cdot) F(c \cdot) \quad(s \in \mathbb{R}, c>0)
$$

is uniformly bounded. Now $F \in \mathcal{F}\left(\mathbf{L}^{\mathbf{1}}(\mathbb{R})\right)$ by (a) of Lemma 5.2, and $\operatorname{sgn}(\cdot)$ is a bounded Fourier multiplier since $X$ is UMD. Hence the claim follows from (c) of Lemma 4.1. 


\section{Proof that $B=A^{1 / 2}$ and compatibility of functional calculi}

Let $-A$ be the generator of a uniformly bounded cosine function Cos on the Banach space $X$, and let $B=\Phi(|t|)$, where $\Phi$ is the functional calculus constructed in Sect. 2. As a first step, let us make sure that the operator $A^{1 / 2}$ is defined by classical theory. This amounts to proving that $A$ is a sectorial operator.

For the following, recall the abbreviation

$$
S_{\omega}:=\{z \in \mathbb{C} \backslash\{0\}|| \arg z \mid<\omega\}
$$

with $\omega \in(0, \pi]$. For background and terminology of sectorial and strip-type operators we refer to [10, Chap. 3].

Lemma 6.1 Let $-A$ be the generator of a cosine function, uniformly bounded by $M \geq 1$, on a Banach space $X$. Then $\sigma(A) \subset \mathbb{R}_{+}$and A satisfies the resolvent estimate

$$
\left\|R\left(\lambda^{2}, A\right)\right\| \leq \frac{M}{|\lambda||\operatorname{Im} \lambda|}(\lambda \notin \mathbb{R}) .
$$

Furthermore, if $A$ is any operator satisfying (6.1), then $A$ is sectorial of angle 0 and $A^{1 / 2}$ is both sectorial of angle 0 and (strong) strip-type of height 0 . More precisely, there is $\tilde{M} \geq 0$ such that

$$
\left\|R\left(\lambda, A^{1 / 2}\right)\right\| \leq \frac{\tilde{M}}{|\operatorname{Im} \lambda|} \quad(\lambda \notin \mathbb{R}) .
$$

Proof The estimate (6.1) follows directly from the representation (1.2). (Note that one has to replace $A$ by $-A$ there and that the formula extends by holomorphy to all $\operatorname{Re} \lambda>0$ ).

By (6.1) we have

$$
\left\|\lambda^{2} R\left(\lambda^{2}, A\right)\right\| \leq M \frac{|\lambda|}{|\operatorname{Im} \lambda|} \quad(\lambda \notin \mathbb{R}) .
$$

Now if $\varphi \in(0, \pi)$ and $\mu \in \mathbb{C} \backslash \overline{S_{\varphi}}$, then there is $\lambda \notin \mathbb{R}$ such that $\lambda^{2}=\mu$ and $\varphi / 2<|\arg \lambda|<$ $\pi-\varphi / 2$. Hence

$$
\|\mu R(\mu, A)\| \leq \frac{M}{|\sin (\arg \lambda)|} \leq \frac{M}{\sin (\varphi / 2)} \quad\left(\mu \notin \overline{S_{\varphi}}\right) .
$$

This proves that $A$ is sectorial of angle 0 . It follows by general theory of (fractional powers of) sectorial operators [10, Chap. 3] that $A^{1 / 2}$ is well-defined and again sectorial of angle 0 . Choose $M^{\prime}$ such that

$$
\left\|\lambda R\left(\lambda, A^{1 / 2}\right)\right\| \leq M^{\prime} \quad(\operatorname{Re} \lambda<0) .
$$

Now, if $\lambda \notin \mathbb{R}$ write

$$
\frac{1}{\lambda-z}-\frac{1}{(-\lambda)-z}=\frac{2 \lambda}{\lambda^{2}-z^{2}} .
$$

Inserting $A^{1 / 2}$ yields

$$
R\left(\lambda, A^{1 / 2}\right)=2 \lambda R\left(\lambda^{2}, A\right)+R\left(-\lambda, A^{1 / 2}\right) .
$$

If $\operatorname{Re} \lambda \leq 0$ we have by (6.2)

$$
\left\|R\left(\lambda, A^{1 / 2}\right)\right\| \leq \frac{M^{\prime}}{|\lambda|} \leq \frac{M^{\prime}}{|\operatorname{Im} \lambda|} .
$$


If $\operatorname{Re} \lambda \geq 0$ then $\operatorname{Re}(-\lambda) \leq 0$ and hence

$$
\left\|R\left(\lambda, A^{1 / 2}\right)\right\| \leq 2\left\|\lambda R\left(\lambda^{2}, A\right)\right\|+\left\|R\left(-\lambda, A^{1 / 2}\right)\right\| \leq \frac{2 M}{|\operatorname{Im} \lambda|}+\frac{M^{\prime}}{|\operatorname{Im} \lambda|}
$$

by (6.3). So the assertion holds with $\tilde{M}:=M^{\prime}+2 M$.

The aim of this section is not only to show that $A^{1 / 2}=B$ (as defined in Sect. 2) but also to prove that the functional calculus $\Phi$ from Sect. 2 is a proper extension of the functional calculi for $A^{1 / 2}$ as a sectorial operator and as a strip-type operator.

Let us recall some definitions and results from [10, Chap. 2]. For $\varphi \in(0, \pi]$ we define

$$
H_{0}^{\infty}\left(S_{\varphi}\right):=\left\{f \in H^{\infty}\left(S_{\varphi}\right)|\exists M, s>0:| f(z) \mid \leq M \min \left(|z|^{s},|z|^{-s}\right)\right\} .
$$

The primary functional calculus for $A^{1 / 2}$ as a sectorial operator is given by

$$
\Psi(f):=\frac{1}{2 \pi i} \int_{\Gamma} f(z) R\left(z, A^{1 / 2}\right) \mathrm{d} z
$$

for $f \in H_{0}^{\infty}\left(S_{\varphi}\right)$, where $\varphi \in(0, \pi)$ and $\Gamma=\partial S_{\varphi^{\prime}}$ with $0<\varphi^{\prime}<\varphi$ being arbitrary. Since these functions are holomorphic, they are determined completely by their restrictions to $\mathbb{R}_{+}$ and we will tacitly perform this restriction whenever it is convenient.

Proposition 6.2 Let $-A$ be the generator of a uniformly bounded cosine function on a Banach space $X$. Let $\Phi$ denote the functional calculus defined in Sect. 2, with $B:=\Phi(|t|)$. Let $\varphi \in(0, \pi)$ and $f \in H_{0}^{\infty}\left(S_{\varphi}\right)$. Then $f \in \mathcal{E}\left(\mathbb{R}_{+}\right)$and $\Phi(f)=\Psi(f)$.

Proof Fix $\varphi^{\prime} \in(0, \varphi)$ and let $\Gamma:=\partial S_{\varphi^{\prime}}, \Gamma_{+}=\mathbb{R}_{+} e^{i \varphi^{\prime}}, \Gamma_{-}:=\mathbb{R}_{+} e^{-i \varphi^{\prime}}$. Then by Cauchy's theorem

$$
\frac{1}{2 \pi i} \int_{\Gamma} f(z) R\left(-z, A^{1 / 2}\right) \mathrm{d} z=0
$$

Hence

$$
\begin{aligned}
\Psi(f) & =\frac{1}{2 \pi i} \int_{\Gamma} f(z) R\left(z, A^{1 / 2}\right) \mathrm{d} z \\
& =\frac{1}{2 \pi i} \int_{\Gamma} f(z)\left[R\left(z, A^{1 / 2}\right)-R\left(-z, A^{1 / 2}\right)\right] \mathrm{d} z \\
& =\frac{1}{\pi i} \int_{\Gamma} f(z) z R\left(z^{2}, A\right) \mathrm{d} z \\
& =\frac{1}{\pi i} \int_{\Gamma_{+}} f(z) z R\left((-i z)^{2},-A\right) \mathrm{d} z-\frac{1}{\pi i} \int_{\Gamma_{-}} f(z) z R\left((i z)^{2},-A\right) \mathrm{d} z \\
& =\frac{1}{\pi} \int_{\Gamma_{+}} f(z)(-i z) R\left((-i z)^{2},-A\right) \mathrm{d} z+\frac{1}{\pi} \int_{\Gamma_{-}} f(z)(i z) R\left((i z)^{2},-A\right) \mathrm{d} z
\end{aligned}
$$




$$
\begin{aligned}
& =\frac{1}{\pi} \int_{\Gamma_{+}} f(z) \int_{0}^{\infty} e^{i z s} \operatorname{Cos}(s) \mathrm{d} s \mathrm{~d} z+\frac{1}{\pi} \int_{\Gamma_{-}} f(z) \int_{0}^{\infty} e^{-i z s} \operatorname{Cos}(s) \mathrm{d} s \mathrm{~d} z \\
& =\int_{\mathbb{R}}\left[\frac{1}{2 \pi} \int_{\Gamma_{+}} f(z) e^{i z|s|} \mathrm{d} z+\frac{1}{2 \pi} \int_{\Gamma_{-}} f(z) e^{-i z|s|} \mathrm{d} z\right] \operatorname{Cos}(s) \mathrm{d} s .
\end{aligned}
$$

It is routine to check that the function

$$
g(s):=\frac{1}{2 \pi} \int_{\Gamma_{+}} f(z) e^{i z|s|} \mathrm{d} z+\frac{1}{2 \pi} \int_{\Gamma_{-}} f(z) e^{-i z|s|} \mathrm{d} z \quad(s \neq 0)
$$

is in $\mathbf{L}^{\mathbf{1}}(\mathbb{R})$. By specializing $X=\mathbb{C}$ and $A=t^{2} \geq 0$ we obtain

$$
\mathcal{C}(g(s) \mathrm{d} s)(t)=f(t) \quad(t \in \mathbb{R}) .
$$

This finally yields $\Psi(f)=\Phi(f)$ as claimed.

Consider now the function $e(z):=(1+z)^{-1}$ and note that

$$
f(x):=e(z)-\frac{1}{1+z^{2}}=\frac{z^{2}-z}{(1+z)\left(1+z^{2}\right)}=\frac{z(z-1)}{(1+z)\left(1+z^{2}\right)} \in H_{0}^{\infty}\left(S_{\varphi}\right)
$$

for any $\varphi<\pi / 2$. Therefore,

$$
\left(1+A^{1 / 2}\right)^{-1}=\Psi(f)+(1+A)^{-1}=\Phi(f)+\left(1+B^{2}\right)^{-1}=(1+B)^{-1} .
$$

This implies that $A^{1 / 2}=B$. Moreover, a look on the construction of the sectorial functional calculus for $A^{1 / 2}$ in [10, Chap. 2] makes it clear that the functional calculus $\Phi$ is a proper extension of it. (By [10, Proposition 1.2.7] one can then conclude that the compatibility for the primary calculi carries over to the unbounded extensions.)

Acknowledgments This article was completed while the author enjoyed a two-month stay at the Institute of Mathematics of the Polish Academy of Sciences in Warsaw and at the Nikolaus-Copernicus University in Torun, Poland. The stay was supported by the EU Marie Curie "Transfer of Knowledge" project "Operator Theory Methods for Differential Equations" (MTKD-CT-2005-030042, TODEQ), and the author is grateful for this support and the kind invitation by J. Zemánek (Warsaw) and Y. Tomilov (Toruñ). The author is also grateful to Bernhard Haak (Bordeaux) who made valuable comments on a previous version of this article.

Open Access This article is distributed under the terms of the Creative Commons Attribution Noncommercial License which permits any noncommercial use, distribution, and reproduction in any medium, provided the original author(s) and source are credited.

\section{References}

1. Arendt, W., Batty, C.J.K., Hieber, M., Neubrander, F.: Vector-Valued Laplace Transforms and Cauchy Problems. Monographs in Mathematics 96. Birkhäuser-Verlag, Basel (2001)

2. Berkson, E., Gillespie, T.A., Muhly, P.S.: Generalized analyticity in UMD spaces. Ark. Mat. 27(1), 1-14 (1989)

3. Bourgain, J.: Some remarks on Banach spaces in which martingale difference sequences are unconditional. Ark. Mat. 21(2), 163-168 (1983)

4. Burkholder, D.L.: A geometrical characterization of Banach spaces in which martingale difference sequences are unconditional. Ann. Probab. 9(6), 997-1011 (1981)

5. Cioranescu, I., Keyantuo, V.: On operator cosine functions in UMD spaces. Semigroup Forum 63(3), 429-440 (2001) 
6. Coifman, R.R., Weiss, G.: Transference methods in analysis. In: Conference Board of the Mathematical Sciences Regional Conference Series in Mathematics, No. 31. American Mathematical Society, Providence (1976)

7. Coifman, R.R., Weiss, G.: Some examples of transference methods in harmonic analysis. In: Symposia Mathematica (Convegno sull'Analisi Armonica e Spazi di Funzioni su Gruppi Localmente Compatti, INDAM, Rome, 1976), vol. XXII, pp. 33-45. Academic Press, London (1977)

8. Fattorini, H.O.: Ordinary differential equations in linear topological spaces II. J. Differ. Equ. 6, 50-70 (1969)

9. Fattorini, H.O.: Uniformly bounded cosine functions in Hilbert space. Indiana Univ. Math. J. 20, 411$425(1970 / 71)$

10. Haase, M.: The Functional Calculus for Sectorial Operators. Number 169 in Operator Theory: Advances and Applications. Birkhäuser-Verlag, Basel (2006)

11. Haase, M.: Functional calculus for groups and applications to evolution equations. J. Evol. Equ. 7, 529554 (2007)

12. Hille, E., Phillips, R.S.: Functional Analysis and Semi-Groups. 3rd printing of rev. ed. of 1957. The American Mathematical Society. XII, 808, vol. XXXI. Colloquium Publications, Providence, p. 1974

13. Keyantuo, V., Vieten, P.: On analytic semigroup and cosine functions in Banach spaces. Stud. Math. 129(2), 137-156 (1998)

14. Miana, P.J.: Vector-valued cosine transforms. Semigroup Forum 71, 119-133 (2005) 